\title{
Aplicación del método praxeológico en el proceso de formación professional
}

\author{
Aplicação do método praxeológico no processo de formação em \\ profissional
}

\section{Application of the praxeological method in the professionals training process}

\author{
Benjamín Barón Velandia (bbaron@ uniminuto.edu) \\ Corporación Universitaria Minuto de Dios - UNIMINUTO \\ Claudia Andrea Betancur Rojas (cbetancur@uniminuto.edu) \\ Corporación Universitaria Minuto de Dios - UNIMINUTO \\ Flor Yolanda Clavijo Alonso (fclavijoal1@uniminuto.edu.com) \\ Corporación Universitaria Minuto de Dios - UNIMINUTO
}

Resumen: El artículo presenta los resultados de un estudio realizado sobre la aplicación del método praxeológico en el proceso de formación de profesionales no licenciados. Se contó con la participación de 60 profesionales que cursaron el seminario Praxeología pedagógica: elaboración diagnóstica desde la praxeología, de la Corporación Universitaria Minuto de Dios - UNIMINUTO Sede Bogotá Presencial. Para agenciar el proyecto de investigación se tuvieron en cuenta los cuatro momentos del método praxeológico (Ver, Juzgar, Actuar y Devolución Creativa), utilizando los cuatro grandes interrogantes que los movilizan y permiten reconocer su interdependencia $y$ simultaneidad. Con la aplicación de los instrumentos, quienes participaron del curso tuvieron la oportunidad no solo de ser agentes que reconstruyen las demás preguntas de manera deductiva, sino que co-construyen nuevas preguntas que caracterizan los momentos del método, para comprender la singularidad del ejercicio analítico de las acciones humanas en relación con los procesos formativos. La investigación aquí presente reconoció el carácter fundamente de la dinámica de las preguntas que constituyen el método praxeológico, llevándolo a un nivel más aplicativo-reflexivo al contexto cotidiano de su enseñanza y aprendizaje y desmarcándolo de la comprensión reduccionista tradicional de la aplicación mecánica de una serie de pasos.

Palabras-clave: método praxeológico; profesionales no licenciados; enseñanza; aprendizaje.

Resumo: O artigo apresenta os resultados de um estudo realizado sobre a aplicação do método praxeológico no processo de formação em profissional. Participaram 60 profissionais do Seminário Praxeologia Pedagógica: Preparação Diagnóstica da

Recebido em: 30/05/2021

Aceite em: 13/08/2021 
Praxeologia, realizado pela Corporação Universitária Minuto de Dios - UNIMINUTO Sede Bogotá. Para a organização do projeto de pesquisa, foram considerados os quatro momentos do método praxeológico (Ver, Julgar, Agir e Devolução Criativa), utilizando quatro grandes questões que os mobilizam e permitem reconhecer sua interdependência e simultaneidade. Com a aplicação dos instrumentos, os participantes do curso deram a oportunidade no terreno de serem agentes que reconstroem as outras questões de forma dedutiva, uma campanha que co-constrói novas questões que caracterizam os momentos do método, para compreender a singularidade. do exercício analítico das ações humanas em relação aos processos de formação. A pesquisa aqui apresentada reconhece o caráter fundamental da dinâmica das questões que constituem o método praxeológico, levandoo a um nível mais aplicativo-reflexivo do contexto cotidiano de seu ensino e aprendizagem e demarcando-o da compreensão reducionista tradicional da aplicação mecânica. estar de passos.

Palavras-chave: método praxeológico; profissional; ensino; aprendiendo.

Abstract: The article presents the results of a study carried out on the application of the praxeological method in the professionals training process. 60 professionals attended the Seminar Pedagogical Praxeology: Diagnostic Preparation from Praxeology, by the Corporación Universitaria Minuto de Dios - UNIMINUTO Sede Bogotá Presencial. For the organization of the research project, the four moments of the praxeological method (See, Judge, Act and Creative Devolution) were taken into account, using four great questions that mobilize them and allow them to recognize their interdependence and simultaneity. With the application of the instruments, those who participated in the course provided the opportunity on the ground to be agents that reconstruct the other questions in a deductive way, a campaign that co-constructs new questions that characterize the moments of the method, to understand the singularity. of the analytical exercise of human actions in relation to training processes. The research presented here recognizes the fundamental nature of the dynamics of the questions that constitute the praxeological method, taking it to a more applicative-reflective level of the everyday context of its teaching and learning and demarcating it from the traditional reductionist understanding of mechanical application. to be of steps.

Keywords: praxeological method; professionals; teaching; learning.

\section{INTRODUCCIÓN}

Durante sus 29 años de formación de profesionales reflexivos y éticamente responsables con la sociedad y las comunidades, la Corporación Universitaria Minuto de Dios - UNIMINUTO ha venido construyendo su impronta en términos pedagógicos y epistemológicos enmarcados en la praxeología, esta, entendida como la necesaria religazón entre teoría y práctica, entre pensar y hacer, con sus respectivas distinciones, pero sin subordinaciones, sin subalternizar la una a la otra, por el contrario, restituyendo

Recebido em: 30/05/2021

Aceite em: 13/08/2021 
el valor fundamental que las dos fuentes singulares de conocimiento poseen desde su lugar de construcción. Esas redes de conversaciones establecidas entre el saber y la acción, mediadas por la reflexividad, le proporcionan al profesional herramientas contextualizadas que posibilitan la identificación de la eficacia de sus acciones e interpelarse por nuevos caminos por construir para la mejora de sus desarrollos profesionales (JULIAO, 2021).

En ese sentido, la praxeología permite configurar los diversos lugares de observación en los que la construcción teórica ha generado un bucle de retroalimentación entre las prácticas y cómo desde los procesos de sistematización de estas, han emergido de nuevas teorías o modificaciones sustancia les a las existentes. Resulta importante distinguir el lugar de construcción de conocimiento en el que se constituyen la prácticas, lo anterior, sin detrimento de las teorías, ya que esta no busca ser teorizada en tal medida que pierda su esencia, o que requiera de un ropaje diferente al que las configura, por el contrario, el corpus de conocimientos que generan estas, tienen por así decirlo, un estatuto propio de reconocimiento, que les brinda una validación en tanto se circunscriben al campo del que subyacen acogiendo sus criterios (BETANCUR; BARÓN-VELANDIA; CELY. 2021)

De acuerdo con lo anterior, para UNIMINUTO es fundamental dar continuidad a los procesos formativos en torno al método praxeológico. Un método que lee su construcción en clave freireriana en la dialéctica: acción-reflexión-reflexión de la acción-nueva, que es el resultado de un proceso metacognitivo persistente del sujeto sobre sus actos cotidianos. De lo que se trata aquí es de llenar de sentidos las acciones humanas y dejar de lado el empoderamiento de las técnicas, lo que durante mucho tiempo se entronizó como la respuesta a todas las problemáticas. En este mismo sentido, las herramientas e instrumentos para realizar los procesos metacognitivos de los actos humanos son muy útiles en tanto contribuyen como mediaciones para mejorar la condición reflexiva, no pueden limitar el genio emergente del pensamiento hábil y creativo del profesional reflexivo, en términos de SCHÖN (1998).

Promover al interior de UNIMINUTO la continuidad del uso del método praxeológico para llegar a la transformación de las acciones humanas mediante reflexión consiente, también se constituye en un propósito importante que permite la

Recebido em: 30/05/2021

Aceite em: 13/08/2021 
permanencia de su impronta académica, científica y humanística. Promover el sentido y el trasfondo de las preguntas que subyacen en las cuatro fases de la praxeología es importante para identificar la dinámica existente entre los pasos del método y los cambios que allí se dan, pero también es fundamental dar a conocer que tras esas etapas existen unas interpelaciones que posibilitan llenar de compresión un método que llevado al extremos sin la dialéctica a la que nos conducen las preguntas puede caer en el vacío de un mecanicismo sin sentido, tal vez eficiente y riguroso, pero carente de sentido y de horizonte humano (MERTINS; GALLE; SILVA, 2020; MERTINS; DA SILVA; ROSA; GÜNTZEL, 2021).

Para JULIAO (2011) es fundamental la construcción de un método praxeológico que trascienda el telos extensivo de los resultados y se instale en la comprensión de los procesos, por ello es vital que la construcción de las preguntas tengan un componente autopoiético, es decir, que las preguntas no se reduzcan a la necia búsqueda de respuestas, sino que generen en aquellas personas que participan de los procesos formativos actitudes de indagación y transformación en la acción consciente, reflexiva y responsable. En otras palabras, que es fundamental conservar y acrecentar los tipos de conocimiento: teórico (saber que), técnico (saber como) y práctico (saber vivir).

En el presente estudio fue importante distinguir dos conceptos que por lo general rondan en el método praxeológico, por un lado, el concepto de fase y por el otro el concepto de momento, cuando hacemos referencia a los pasos de la praxeología. Cuando en este proyecto nos referimos al Ver, Juzgar, Actuar y Devolución Creativa, lo que queremos entender es que cada una de estas palabras, se configuran como momentos, estados de la acción humana, en el que el sujeto se sumerge para tomar decisiones basadas en hechos sobre lo que hace en tu cotidianidad. Razón por la cual, no se deben interpretar como pasos prerrequisitos para abordar la implementación del método, tal vez el único que podemos reconocer como punto de partida para la reflexión, es el momento del VER, por constituirse en ese instante de contemplación del fragmento de la realidad, del habitar esa primera pregunta, es por ello por lo que, la imagen de la espiral es fundamental para comprender la simultaneidad e interdependencia que religan los momentos en favor de la transformación del sujeto a través del desarrollo del método. (JULIAO, 2020)

Recebido em: 30/05/2021

Aceite em: 13/08/2021 
De acuerdo con lo anterior, la aplicación de los momentos de método praxeológico nos invita a una compresión compleja del análisis de nuestras acciones, por ello no resulta viable separarlos, aislarlos o desligarlos el uno del otro y mucho menos del agente quien es el poseedor de la consciencia de sí. Por el contrario, es en la simbiosis, en la mixtura entre ellos en los que nutrimos la experiencia del sujeto. Es fundamental reconocerlos como puntos de referencia o lugares de observación transitorios desde donde abordamos la problemática en cuestión y desde donde emergen también nuevas comprensiones o posibles resoluciones a los interrogantes que nos vamos planteando.

Ahora bien, veamos un poco en sentido más detenido las grandes preguntas que los momentos contienen y empecemos a desarrollar metodológicamente cómo todo ello permite la transformación de las prácticas del agente directo y cómo de manera indirecta termina transformando escenarios y otros agentes que lo transitan.

\section{LOS MOMENTOS DEL MÉTODO PRAXEOLÓGICO}

La primera pregunta a la que nos invita el método praxeológico desde el VER es interpelarnos ¿Qué sucede con mi práctica? interrogante que está sumergido en actitudes de exploración y de análisis/síntesis, tipificado principalmente por el desarrollo cognitivo en el que profesional/praxeólogo colecciona la información sobre la práctica, la vuelve objeto de investigación para asirla en un contexto conformado por las vivencias y experiencias que nutre esta configuración que el agente hace "elementos, racionalidad, desarrollo en el tiempo y eficacia en función de los objetivos, a veces mal o incluso no formulados o, al contrario, claramente definidos" (JULIAO, 2011, p.36).

Preguntas emergentes que contribuyen al matiz del momento metodológico del VER

¿Quién hace qué? ¿Por quién lo hace? ¿Con quién? ¿Dónde? ¿Cuándo? ¿Cómo? y ¿Por qué lo hace?

La segunda pregunta que emerge desde el JUZGAR es ¿Qué puede hacerse con mi práctica? de manera simultánea y en la acción en la que nos interpelábamos por ¿Qué sucede con mi práctica? se empieza a tejer la reacción hermenéutica en la que el

Recebido em: 30/05/2021

Aceite em: 13/08/2021 
"profesional/praxeólogo examina otras formas de enfocar la problemática de la práctica, se ubica en otros lugares de observación que le permiten agenciar acciones teorías, siempre buscando comprender sus prácticas, construir una cosmovisión de sí y desarrollar empatía para participar y comprometerse con ella" (JULIAO, 2011, p.38).

La tercera pregunta proviene del JUZGAR y se religa con las anteriores porque pretende dar sentido al ¿Qué sucede con mi práctica? y a la primera reacción por intentar saber ¿Qué puede hacerse con mi práctica? y se ubica en el plano de ¿Qué hacemos en concreto? es decir, de concebir el problema, pasamos a la elaboración propia de comprensión de la práctica, parque llevar a cabo acciones programáticas que permitan reconocer las transformaciones surgidas de transgredir la práctica, entrar en diálogo con autores o teorías, para tener herramientas que contribuyan en el establecimiento de acciones puntuales que eviten el círculo vicioso de hacer lo mismo y querer encontrar resultados diferentes, por el contrario, lo que aquí se busca es la acción plástica del agente sobre su intervención, que se permita habar de círculo virtuoso en el que la teoría del rejuvenecimiento que le da la reflexión sobre las acciones, posibilita la mejora de las capacidades de interpretar e intervenir la realidad en la acción inmediata y sutil.

La cuarta pregunta que está latente todo el tiempo, porque en realidad baña todo el proceso del método es ¿Qué aprendemos de lo que hacemos? durante cada uno de los momentos siempre estuvo presente esa necesidad de estar aprendiendo de estar incorporando los entendidos a lo largo de todo lo que se hace, es lo que entendemos como la Devolución Creativa, un momento que Juliao (2011, p.43) plantearía de la siguiente manera:

Se trata de un acto existencial autogenerador de teoría a partir de la experiencia, que requiere ser sacado a la luz, a través de un proceso mayéutico que le permita objetivar dicha experiencia, formalizarla y entrar en el orden del discurso (así se corra el riesgo de deformar la experiencia): es una recuperación de la praxis por el logos (inter y autoestructurante) se trata del diálogo establecido entre practicantes y prácticas, que permite desarrollar más y mejor los conocimientos de éstos.

Con estos conceptos aclarados y presentadas las preguntas que nos van a servir como categorías de colección de nuevas preguntas emergidas por el grupo de los 108 participantes, avancemos en sentido estricto por los procesos de implementación del método en los cuatro momentos, mediados por las co-construcciones de los nuevos 
agentes quienes tendrán también la oportunidad de objetivar la realidad desde su elaboración de mundo "subjetivación".

\section{METODOLOGIA}

\section{Diseño}

La presente investigación se fundamenta en el paradigma cualitativo-descriptivo, ya que presenta los resultados de un estudio realizado sobre la aplicación del método praxeológico en el proceso de formación de un grupo de profesionales no licenciados que cursaron el seminario Praxeología pedagógica: elaboración diagnóstica desde la praxeología.

\section{Participantes}

La investigación tuvo como contexto de aplicación la Corporación Universitaria Minuto de Dios - UNIMINUTO Sede Bogotá Presencial en Bogotá, Colombia. La selección de la muestra fue por conveniencia entre los grupos de profesionales no licenciados (se entiende en el contexto colombiano a los profesionales no licenciados como profesionales de la educación, que ejercen la docencia sin poseer un título de licenciados o tener formación pedagógica, pero que a través del decreto 1278 de 2002 se encuentran habilitados para ejercer la docencia en los niveles de básica secundaria y media en Colombia (MEN; 2002) para mejorar las condiciones de calidad de los profesionales no licenciados el Gobierno colombiano generó una estrategia para brindarle formación pedagógica a estas personas y que se pudieran vincular al sistema educativo y brindar un servicio de calidad).

Para agenciar el proyecto de investigación se tuvieron en cuenta los cuatro momentos del método praxeológico (Ver, Juzgar, Actuar y Devolución Creativa) de acuerdo con la explicación conceptual anteriormente planteada.

\section{Instrumentos}

Para la aplicación del método praxeológico en el proceso de formación de un grupo de profesionales se utilizaron principalmente 7 Fichas (JULIAO, 2011) con sus

Recebido em: 30/05/2021

Aceite em: 13/08/2021 
respectivos propósitos, lo que permitió agrupar de manera directa las respuestas de quienes hicieron parte de la investigación:

Ficha $\mathrm{N}^{\circ}$ 1: HAGO MEMORIA DE MI EXPERIENCIA VIVIDA: AUTOOBSERVACIÓN

Propósito: Escribir el relato autobiográfico de mi experiencia o práctica como persona y profesional (y de ser el caso como maestro), identificando los acontecimientos claves más desatacados (hitos fundamentales) en mi itinerario de vida profesional.

Ficha $N^{\circ}$ 2: HAGO MEMORIA DE MI EXPERIENCIA - SOY COMPETENTE PARA...

Propósito: ¿Reflexionar y analizar sobre las competencias que poseo, en diversos niveles de la vida y el aprendizaje, y que se convierten en mis "herramientas de trabajo profesional" Soy competente para...

Ficha $\mathrm{N}^{\circ}$ 3: COMPRENSIÓN DE LOS CONCEPTOS PEDAGÓGICOS FUNDAMENTALES

Propósito: Sintonizar/unificar los conceptos y entendidos que se tendrán como derroteros para el desarrollo de la implementación del método.

Ficha $\mathrm{N}^{\circ}$ 4: DESCRIBO MI PRÁCTICA PROFESIONAL

Propósito: Reflexionar sobre nuestras prácticas profesionales, a partir del describir, analizar y discernir una práctica concreta (situada, contextualizada en el tiempo y el espacio) en el ejercicio profesional.

Ficha $N^{\circ}$ 5: ME PREGUNTO POR MI PRÁCTICA PROFESIONAL

Propósito: Analizar la práctica elegida y descrita, a partir de la identificación de sus fortalezas y de las inconformidades que encontramos en ella.

Ficha $N^{\circ}$ 6: DISCIERNO MI PRÁCTICA PROFESIONAL

Propósito: Discernir aquellas situaciones que nos interrogan sobre nuestras prácticas profesionales y que son una oportunidad para reconocer las posibilidades de un crecimiento personal y de una conversión profesional.

Ficha $N^{\circ}$ 7: REPLANTEO MI PRÁCTICA PROFESIONAL

Propósito: Integrar lo aprendido hasta el momento en el ejercicio de revisión crítica dentro de mi proyecto de vida y desarrollo personal y profesional.

Recebido em: 30/05/2021

Aceite em: 13/08/2021 
La manera como se aplicaron las fichas fue la siguiente:

Por tratarse de un tema tan íntimo, porque se trata de hacer un ejercicio desde dentro de la condición personal, volver sobre ciertos momentos de la vida que cada uno seleccionó "hitos", aquí el docente recibió orientación en términos metodológicos generales sobre cada uno de los ejes de las guías. Quien guiaba el ejercicio se limitó a un nivel de acompañamiento, es decir, no leyó lo que cada uno de los 60 participantes iba escribiendo. Realmente asumió el papel de guía metodológico. Asesoró la resolución de inquietudes que las preguntas de las guías tenían en relación con los conceptos que allí se encontraban. En algunos momentos después de generar las claridades de las fichas, convidó a quienes participaban del ejercicio a que cambiaran de espacios, que buscaran un lugar en el que se sintiera más libres, con tranquilidad, sin presiones, ni sensaciones de cumplimiento con alguien más que con cada uno de nosotros.

Sin lugar a duda, existieron momentos en los que se sintió mayor tensión, por ejemplo, cuando se interpelaba por el replanteamiento de ciertas prácticas profesionales en las que el mismo sujeto puso en observación, a muchos les costó imaginarse en un lugar diferente de trabajo, o haciendo algo que realmente le guste, pero que no era tan aceptado por la mayoría como era lo que actualmente hacían. Como profesionales, muchos pertenecían al campo de la ingeniería y las ciencias administrativas, lo que en su plan de vida no se imaginaron nunca estar trabajando como profesores.

La aplicación y desarrollo de las fichas realmente generaba otra atmosfera. Era evidente la inversión de los roles, el reconocimiento de cada uno de los participantes en ese espacio de interaprendizajes. Para algunos participantes que tenían mucha experiencia como docentes, asumir el rol de estudiante, seguir instrucciones y consultar sobre dudas con los conceptos o la metodología, fue realmente complejo y desafiante. En un principio, muchos dudaron en continuar con el ejercicio y se les dificultó hablar de sus prácticas pedagógicas en aulas.

Las personas que participaron siempre conservaban sus fichas, con sus avances. Poco a poco la dinámica de acompañamiento se fue haciendo mínima, porque una vez se terminó de explicar las fichas y su propósito, cada participante fue realizando un ejercicio autónomo de encuentro con su instante o instantes preferidos. Muchos 
avanzaron en descripciones de personas que significaron mucho en sus vidas y que se les volvieron importantes. Algunos, decidieron ahondar en conceptos que alguna ficha tenía. Otros, fueron muy lacónicos en las descripciones que las fichas solicitaban.

\section{RESULTADOS}

\section{Caracterización del grupo}

El grupo de profesionales que participó de la investigación, Figura 1, se caracterizó principalmente por tener una alta participación de Mujeres (33) y 27 Hombres. Reconociendo que fue por voluntad propia de los participantes, es evidente la motivación de las mujeres por llevar a cabo un ejercicio de metacognición de su quehacer docente.

Figura 1. Caracterización por género de quienes participaron

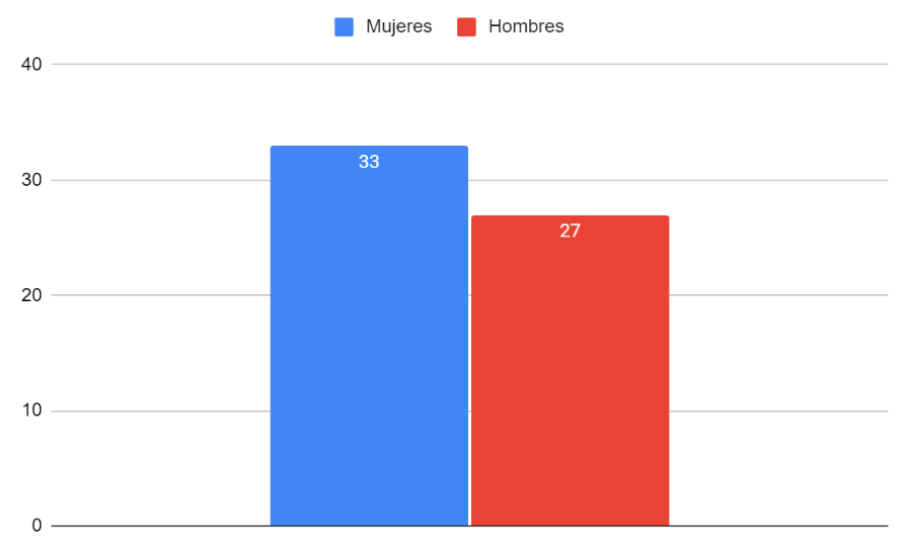

Fuente: Barón; Betancur; Clavijo, 2021.

En relación con las preguntas que abarcaron la comprensión del método praxeológico, a continuación, se hace la síntesis de los resultados de las participaciones de los profesionales y las propuestas que surgen durante el desarrollo del análisis de las fichas para la transformación o la adaptación de nuevas prácticas pedagógicas a través del ejercicio de reflexividad que se condujo a través de las 7 fichas:

Cuadro 1 - Síntesis de aprendizajes que dejó el método.

Recebido em: 30/05/2021

Aceite em: 13/08/2021 


\begin{tabular}{|c|c|}
\hline Preguntas & Aprendizajes que dejó el método \\
\hline $\begin{array}{c}\text { ¿Qué sucede con mi } \\
\text { práctica? }\end{array}$ & $\begin{array}{l}\text { - No es hacer las cosas mecánicamente } \\
\text { - Implica una consciencia del pasado y del futuro } \\
\text { - Es como mirarse en un espejo, pero con la posibilidad de reorientar } \\
\text { los hechos. } \\
\text { - Descubrí mi historia familiar latente. } \\
\text { - Existe paradigmas en mí que no conocía. } \\
\text { - Hay una presencia de personas que no soñé ser. } \\
\text { - No podemos mentirnos a nosotros mismos sobre la realidad de lo que } \\
\text { hacemos y el porqué lo hacemos. } \\
\text { - Se puede aplicar a la vida cotidiana para mejorar lo que hacemos y } \\
\text { como vivimos. } \\
\text { - Es una confrontación con nuestras elecciones y decisiones de vida. } \\
\text { - Se requiere mucha madurez para hacer el ejercicio con alguien } \\
\text { diferente a uno mismo. }\end{array}$ \\
\hline $\begin{array}{l}\text { ¿Qué puede hacerse } \\
\text { con mi práctica? }\end{array}$ & $\begin{array}{l}\text { - Buscamos modelos para seguir, más que diseñar para dejarle a los } \\
\text { demás. } \\
\text { - Después de la autoobservación inmediatamente queremos cambiar } \\
\text { ciertas conductas y prácticas que tenemos en todas las dimensiones } \\
\text { personal, familiar, laboral. } \\
\text { - En un principio desesperanza, porque siempre décimos que lo que } \\
\text { desconocemos es mayor a lo que conocemos, pero cuando somos } \\
\text { conscientes de ello, realmente sabemos lo que significa. }\end{array}$ \\
\hline Ista & $\begin{array}{l}\text { - Transformar y cambiar los rasgos paradigmáticos laborales, } \\
\text { familiares, sociales con los que no nos sentimos identificados y que } \\
\text { utilizamos como mecanismos de defensa. }\end{array}$ \\
\hline $\begin{array}{c}\text { ¿Qué hacemos en } \\
\text { concreto para mejorar } \\
\text { mi práctica? }\end{array}$ & $\begin{array}{l}\text { - Es una voz de esperanza después del ejercicio de catarsis que se tiene } \\
\text { con las dos primeras Fichas. } \\
\text { - Fijarnos metas reales después de revisar nuestras historias de vida de } \\
\text { manera individual. } \\
\text { - La posibilidad de concretar acciones y hacerles seguimiento. } \\
\text { - Hacer cosas que siempre quise hacer } \\
\text { - Establecer "acciones programáticas" genera en los sujetos deseos de } \\
\text { "progreso" de superación de las dificultades. } \\
\text { - Dejar de planear en abstracto y postergar en la realidad, para pasar a } \\
\text { la construcción de un proyecto de vida con mayor sentido humano. }\end{array}$ \\
\hline
\end{tabular}




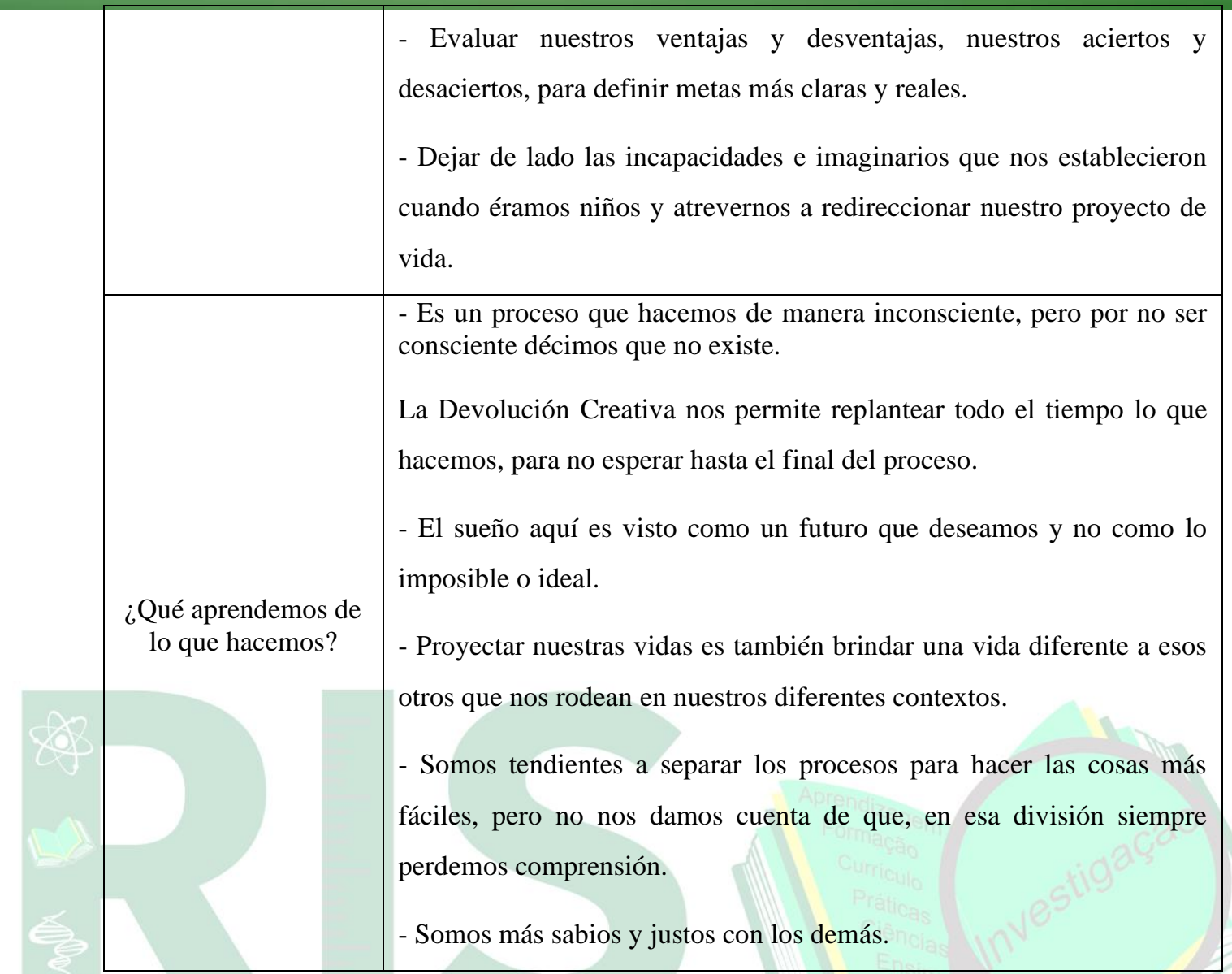

Fuente: Barón; Betancur; Clavijo, 2021.

De acuerdo con das elaboraciones anteriores, es importante destacar que la distancia existente entre las teorías y los preconceptos que tienen sobre el método praxeológico los profesionales no son determinantes para que la comprensión de los momentos de este se vuelvan un obstáculo para el aprendizaje (PINEDA; OROZCO, 2016). Prueba de ello son los "aprendizajes que les dejó el método" Que sus construcciones conceptuales permiten identificar que lo valioso del método trasciende su correcta aplicación según el paradigma del cual emergen en su totalidad, sino como lo plantea ESCUDERO (2020) que existe la posibilidad de generar paradigmas adaptativos, que permitan su cohabitación, en el que el fin último, es decir, comprender la realidad para modificarla, sea superado por la dialéctica entre la praxis y la racionalidad cognitiva (JIMÉNEZ-HERNÁNDEZ; SANCHO-REQUENA; SÁNCHEZFUENTES, 2021). 
De otra parte, al leer el conjunto de aprendizajes que les dejó a los profesionales el método praxeológico, se hace evidente, como lo plantean JULIAO; BARÓN (2013) y BARON (2017) que hacer praxeología es una cuestión vital, porque comprende al sujeto en su multidimensionalidad sin establecer dualismos o binarismos que desliguen la profesión, del ser vivo, que siente, que piensa, que ama, porque trasciende la cultura científica, que no se reduce a un acontecimiento académico, sino que se religa con los demás contextos sociales, comunitarios, laborales, entre otros (GIROUX, 2016). En este orden de ideas, nos invita a una revisión permanente y consciente de nuestras acciones humanas concretas en relación con lo que soñamos ser y no lo que otros sueñan que seamos (VARÓN ARCINIEGAS, V.; MARTÍNEZ URBANO; LEÓN; BARÓNVELANDIA, 2020)

Es considerable que desde una perspectiva más social el método praxeológico sea aceptado como un método para hacer catarsis por su efecto terapéutico, tal y como lo plantean los y las participantes del proyecto cuando asumen posturas de reconocimiento de las debilidades y de las fortalezas, cuando se permiten hacer el ejercicio de introspección de uno de sus hitos y ahondar en la escritura narrativa a modo de viaje contemplativo y se reconstruyen momentos que dejan aflorar sentimientos y emociones "difícilmente descriptibles por la racionalidad cognitiva solamente" (HARGREAVES; FULLAN,2014).

\section{CONCLUSIONES}

Como colofón, es importante destacar que con el ejercicio de aplicación del método praxeológico se logró identificar en las conversaciones y comentarios de los participantes que existe una fuerte influencia de la fenomenología, en tanto que hace un retorno al mundo de la vida y a las cosas, ese volver a la práctica para reinterpretarla y resignificarla en los contextos de análisis, sin reducirla a las estructuras institucionalizadas. En su apertura al mundo de la vida encuentra las grandes dificultades de hacer de los métodos instrumentos sistematizados al extremo, que terminan por volverse inoperantes, porque no existen realidades que se adapten a ellos, cuando tiene que ser todo lo contrario, por referenciar nuevamente la plasticidad.

Recebido em: 30/05/2021

Aceite em: 13/08/2021 
En las diversas conversaciones sostenidas con las / los participantes, se reconoció una postura teleológica en el método, lo que permite fijar en el horizonte no la meta o el punto de llegada, sino el camino, ese fin último en el que descansan los sueños y las aspiraciones de las personas que buscan sentidos diversos a sus existencias. La religazón que nuestros participantes hacen del método como una forma de asumir el reto de vivir y de ser en el mundo, también se ve claramente iluminada por la permanente reflexión del hacer cotidiano que proyecta sus existencias.

Los resultados de la investigación permiten identificar en la aplicación del método praxeológico un escenario de reconocimiento incondicional de las diversas fuentes de conocimiento como legítimas, con el que se amplían las miradas hacia sociedades más justas y equitativas, en las que el respeto por las comprensiones y los lugares de construcción se constituirán en escenarios de diálogo y no en lugares de subalternización de los seres humanos, las especies y los territorios.

Con la experiencia vivida por el equipo de investigación, también se concluye que la aplicación de los momentos del método praxeológico a través de las fichas en espacios de intimidad como los que brinda una de las tantas formas del método praxeológico para auto reflexionar sobre las prácticas, se pueden convertir en estrategias institucionales que fomenten otras dinámicas de reencuentro consigo mismo y con los demás, gestionando cambios, círculos virtuosos, reencontrar frecuentemente la pasión de lo que se hace a diario desde lo profesional, sin detrimento de lo humano de las demás dimensiones que por lo general son distanciadas por estimarse "subjetivas" y no dignas de aparecer en la escena laboral.

Como conclusión colectiva de los participantes y del equipo de investigación se pudo reconocer que el ejercicio de reflexión continua sobre las prácticas pedagógicas, permiten identificar la importancia de capacitarse de manera continua sobre sus áreas de desempeño para enfrentar los grandes desafíos de la educación en el contexto nacional e internacional, también es necesario capacitarse en procesos de formación humana, psicosociales, entre otros, que coadyuven al desarrollo multidimensional de las/os docentes lo que permitirá un mejor desempeño de la profesión y óptimas relaciones interpersonales.

Recebido em: 30/05/2021

Aceite em: 13/08/2021 


\section{REFERENCIAS}

BARÓN-VELANDIA, B. Conversaciones (prácticas vitales en educación superior). Bogotá: Aula Humanidades. 2017.

BETANCUR ROJAS, C., BARÓN-VELANDIA, B., \& CELY BETANCOURT, B. Praxeological Practices. Praxis Pedagógica, 21(28), 1-4. 2021.

CARR, WILFRED; STEPHEN KEMMIS. Teoría crítica de la enseñanza. La investigación acción en la formación del profesorado. Barcelona: Martínez Roca. 1988.

ESCUDERO, J. Un cambio de paradigma en la formación continuada del profesorado: escenario, significados, procesos y actores. Revista Qurriculum, 33, 97125. 2020.

GARCÍA; L. Praxis del desarrollo profesional del docente en escuelas exitosas del Departamento de Educación de Puerto Rico (Tesis Doctoral). Universidad de Puerto Rico, Rio Piedras, Puerto Rico. 2020.

GIROUX, H. «Cultural Studies, Publica Pedagogy and the Responsabilities of Intellectuals». Communication and Cultural Studies, 1(1), 59-79. 2016.

HARGREAVES, A. Y FULLAN, M. Capital profesional. Madrid: Morata. 2014.

JIMÉNEZ-HERNÁNDEZ, D., SANCHO-REQUENA, P. \& SÁNCHEZ-FUENTES, S. Estudio acerca de las opiniones del profesorado universitario en la Región de Murcia sobre la formación de métodos activos. Revista Electrónica Interuniversitaria de Formación del Profesorado, 24(2),113-124. 2021.

JULIAO, C. El enfoque praxeológico. Bogotá: Ed. Uniminuto. 2011.

JULIAO VARGAS, C. La investigación praxeológica. Praxis Pedagógica, v. 20, n. 26, p. 117-148, 3 fev. 2020.

JULIAO VARGAS, C., \& BARÓN VELANDIA, B. La praxeología: otra forma de experimentar la vida. Praxis Pedagógica, 13(14), 141-145. 2013.

MEN. Ministerio de Educación Nacional, 2002. Disponible en:

https://www.mineducacion.gov.co/1621/articles-86102_archivo_pdf.pdf Acesso en: 1 junio. 2019.

MERTINS, S; DA SILVA, C; ROSA, M; GÜNTZEL RAMOS, M. Preguntas de los estudiantes para la enseñanza de las ciencias: un medio para identificar problemas conceptuales. Revista Insignare Scientia - RIS, vol. 4, no. 3, pág. 41-57, 3 de marzo 2021.

MERTINS; Simone. GALLE, Lorita Aparecida Veloso; SILVA, Carla Melo da. Pesquisa como princípio educativo: contribuições das perguntas dos estudantes para a aprendizagem de Química. Revista Insignare Scientia -RIS, v. 3, n. 3, p. 190 a 207, nov. 2020

PINEDA MARTÍNEZ, E.; OROZCO PINEDA, P. El currículo praxeológico como interés de conocimiento emancipatorio. Praxis Pedagógica, v. 16, n. 18, p. 11-25, 1 fev. 2016.

Recebido em: 30/05/2021

Aceite em: 13/08/2021 


\section{Edição Especial: Pesquisa na Pós-Graduação em Ensino de Ciências}

Vol. 4, n. 5. 2021

ISSN: $2595-4520$

SCHÖN, D. El profesional reflexivo. Cómo piensan los profesionales cuando actúan. Barcelona: Paidós. 1998.

VARÓN ARCINIEGAS, V.; MARTÍNEZ URBANO, S.; LEÓN NIETO, Y.; BARÓNVELANDIA, B. Pedagogía de la alteridad: entre la educación como práctica social y el ejercicio intelectual. Revista Insignare Scientia - RIS, v. 3, n. 2, p. 484-508, 25 ago. 2020.

Recebido em: 30/05/2021

Aceite em: 13/08/2021 